\title{
EFFECT OF FUEL TYPE ON FLAME IGNITION BY TRANSIENT PLASMA DISCHARGES
}

\author{
Jianbang Liu ${ }^{1,2}$, Fei Wang ${ }^{1}$, Long C. Lee ${ }^{1}$, Paul D. Ronney ${ }^{2}$, and Martin A. Gundersen ${ }^{1, *}$ \\ Department of Electrical Engineering ${ }^{1}$ and Department of Aerospace and Mechanical Engineering ${ }^{2}$ \\ University of Southern California, Los Angeles, CA 90089
}

\begin{abstract}
Rise and delay times of mixtures of methane, propane, n-butane, iso-butane and isooctane mixed with air ignited by transient plasma discharge were investigated and compared with spark discharge ignition. Multi-ignition site effect and high electron energy are suggested to contribute to shortening of rise and delay times.
\end{abstract}

\section{INTRODUCTION}

The spark discharge has been the preferred ignition source for most propulsion and automotive combustion engines for over 100 years. It has many advantages including simplicity, low cost, size and weight, and it produces sufficiently high temperatures to partially dissociate and ionize most fuel and oxidant molecules. Nevertheless, it has numerous limitations including limited discharge size, necessity for intrusive supporting electrodes, and low "wall-plug" efficiency (ratio of energy deposited in the gas to electrical energy consumed to produce the discharge.) Consequently, ignition by laser sources has been pursued ${ }^{1,2}$. Still, laser sources present practical difficulties including optical access, extremely low wall-plug efficiency, and extremely high optical intensities needed to induced gas breakdown that makes controlling the discharge location and energy difficult.

This work utilizes transient plasma or pulsed corona discharges for flame ignition. These discharges comprise the early transient phases of high-voltage electric discharges that precede the quasi-steady arc phase, which persists until the electric field is removed. Pulsed corona discharges are terminated before the arc phase begins. Such discharges cannot be produced by conventional ignition systems. Moreover, the ignition method examined here is entirely different from "plasma jets" ${ }^{1}$ whereby spark are discharged in a prechamber (sometimes containing different reactants) and radials/ions thereby generated expand via gasdynamics into the main combustion chamber. Our system has no prechamber or auxiliary reactants.

Flame ignition by pulsed corona discharge or by similar high voltage (typically $10 \mathrm{~s} \mathrm{KV}$ ) short pulse width (typically 10-100 ns) has been reported ${ }^{3-10}$. In this paper, combustion performance of various fuels including methane, propane, iso-butane, n-butane and iso-octane mixed with air and ignited by pulsed corona discharge will be presented and compared with spark discharge ignition. Possible mechanism will be suggested and discussed.

\section{EXPERIMENT SETUP AND RESULTS}

Experiments were carried out in a test cylinder of 2.5" ID and 6" length with a high voltage electrode along central axis as anode and a grounded outer cylinder as cathode. A pulse power generator provided electric pulses with 60 $\mathrm{KV}$ maximum voltage. $1 \mathrm{~J}$ maximum pulse energy and 100ns pulse width. High voltage pulse generator consisted of thyratron, Blumlein transmission line and transformer. The special electrode structure and short pulse width are intended to make the discharge a pulsed corona discharge with no arc discharge involved. For comparison, a spark discharge gap was used which has a $1 \mathrm{~mm}$ separation and located either at the center or on the end plate of the combustion chamber.

A pressure transducer (Omega PX4201) with $0.2 \mathrm{~ms}$ response time was adopted along with a digital oscilloscope (Tektronix TDS 420A) to measure pressure waveform. Delay time (defined as the time laps between trig and 
pressure rises to $10 \%$ of its total pressure rise), rise time (defined as the time laps between pressure rises to $10 \%$ and $90 \%$ of its total pressure rise) and peak pressure were measured from pressure waveforms.

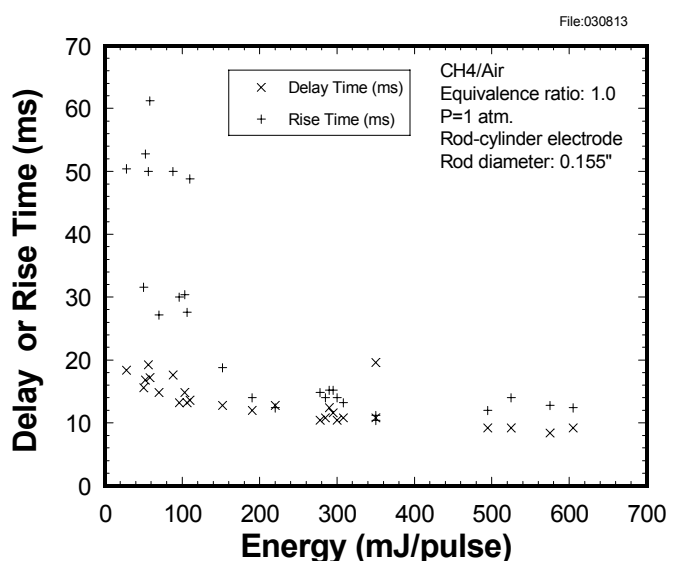

Fig.1 Delay and rise times versus energy. $\mathrm{CH}_{4}$ /Air, Equivalence ratio:1.0

Figurel shows a typical delay and rise times versus energy of stoichiometric $\mathrm{CH}_{4} / \mathrm{Air}$ mixture with a rod-cylinder electrode (rod diameter: 0.155"). As energy increasing, delay time decreases slightly but rise time changes rapidly with increasing energy. At low energy, rise times are longer and scatter. There is an energy value (e.g. $350 \mathrm{~mJ}$ in Fig.1) above this value, rise time has its smallest value and almost constant, below this value, rise times are relatively long and scatter. This is "optimum energy" because it produces shortest stable rise time with lowest energy. Optimum energy is higher for leaner mixtures.

Figures $2 \mathrm{a}, \mathrm{b}$ show delay time and rise time, respectively, for corona-ignited and sparkignited $\mathrm{CH}_{4}$-air flames at varying equivalence ratios. Single pin electrode was used for corona discharge. Therefore, both pulsed corona and spark discharge had only one ignition site. In addition, energies were chosen the same for both types of discharges. In this identical ignition site number and identical energy case, delay time of pulsed corona ignition is significantly lower than that of spark discharge (Fig. 2a), implying there must be another factor, which can significantly affects delay time. As for rise time, if ignition site numbers and energy are the same, there is no big difference between corona and spark discharges (Fig.2b). But if multi-ignition site electrode are used, rise time can be significantly shortened. For example, 4 ring x 2 pin electrode (Fig.2b) can shorten rise time by a factor of 3 compared to spark discharge.

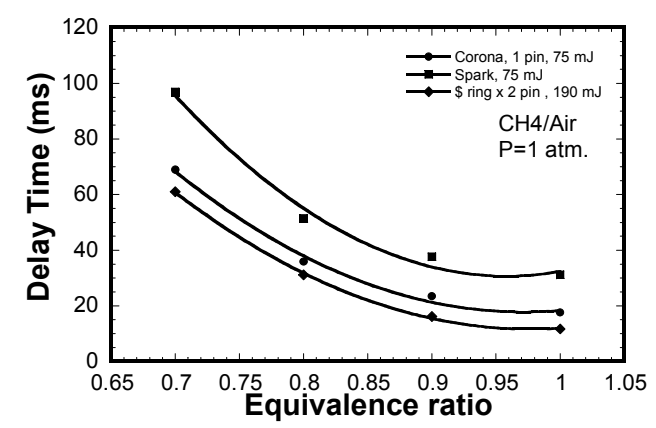

Fig.2a Delay time versus equivalence ratio for pulsed corona with $1,4 \times 2$ electrode and spark discharges.

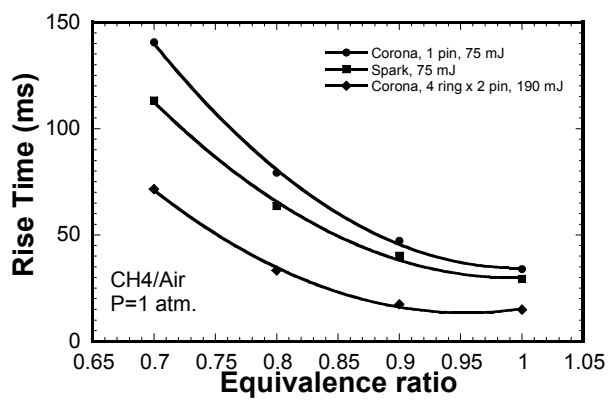

Fig.2b Rise time versus equivalence ratio for pulsed corona with 1, 4 × 2 electrode and spark discharges

Figure 3 shows pressure effects on rise time for propane-air mixtures. Atmospheric pressure data show trends similar to methane-air, with rise times for corona discharges still typically $3 \mathrm{x}$ smaller than for sparks, although the increase in rise time as equivalence ratio decreases occurs at higher equivalence ratio. For higher pressures, the advantage of corona discharges vs. spark discharges is maintained to lower equivalence ratios.

To examine chemical effects, n-butane and iso-butane fuels were compared. These have nearly identical transport properties, heating values and burning velocities but n-butane has 
four secondary $\mathrm{C}-\mathrm{H}$ bonds and no tertiary $\mathrm{C}-\mathrm{H}$ bonds whereas iso-butane has no secondary $\mathrm{C}-\mathrm{H}$ bonds and one tertiary $\mathrm{C}-\mathrm{H}$ bond. Tertiary $\mathrm{C}-\mathrm{H}$ bonds are weaker than secondary bonds, which are weaker than primary bonds; effects of bond strengths on ignition of non-ionized mixtures are well known ${ }^{1}$. Figure 4 shows that spark and corona discharges show the same trends with changing equivalence ratio even though these trends are somewhat different for n-butane vs. iso-butane; either n-butane or iso-butane may yield lower rise times depending on equivalence ratio.

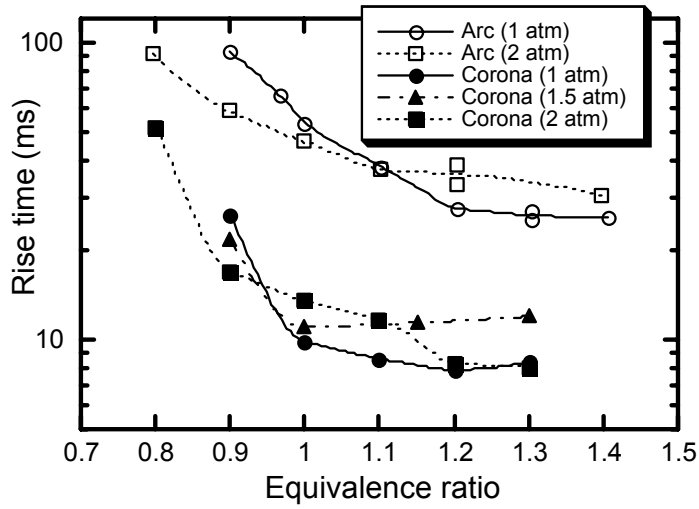

Fig.3 Rise times of corona-ignited (plain electrode) and spark-ignited (central ignition) $\mathrm{C}_{3} \mathrm{H}_{8} /$ Air flames at 1 atm. as a function of equivalence ratio and pressure

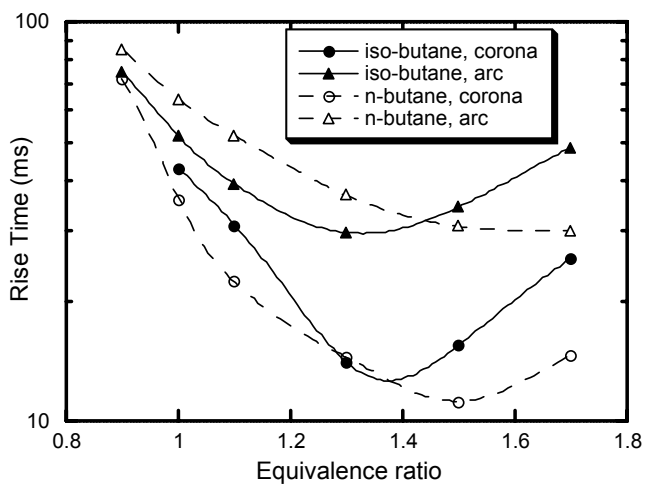

Fig.3 Rise times of corona-ignited (plain electrode) and spark-ignited (central ignition) n$\mathrm{C}_{4} \mathrm{H}_{10} /$ Air and iso- $\mathrm{C}_{4} \mathrm{H}_{10} /$ Air flames at $1 \mathrm{~atm}$. as a function of equivalence ratio. Delay times (not shown) were nearly identical for all 4 cases.

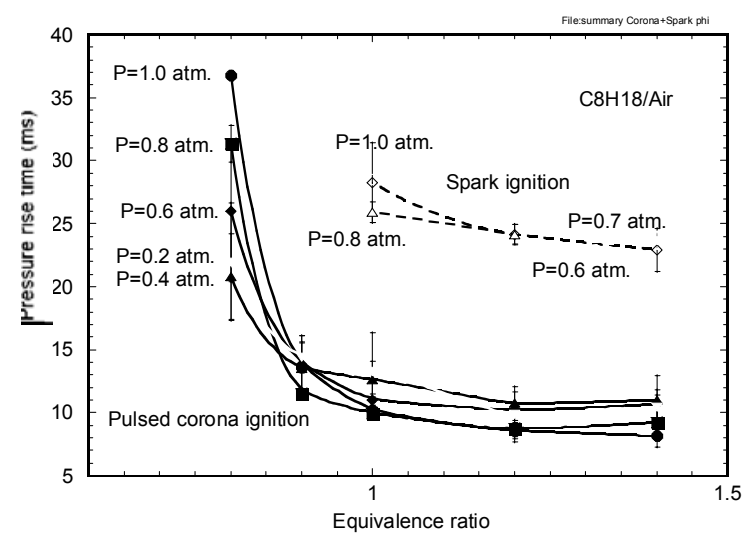

Fig.5 Rise time versus equivalence ratio of iso$\mathrm{C}_{8} \mathrm{H}_{18} /$ Air

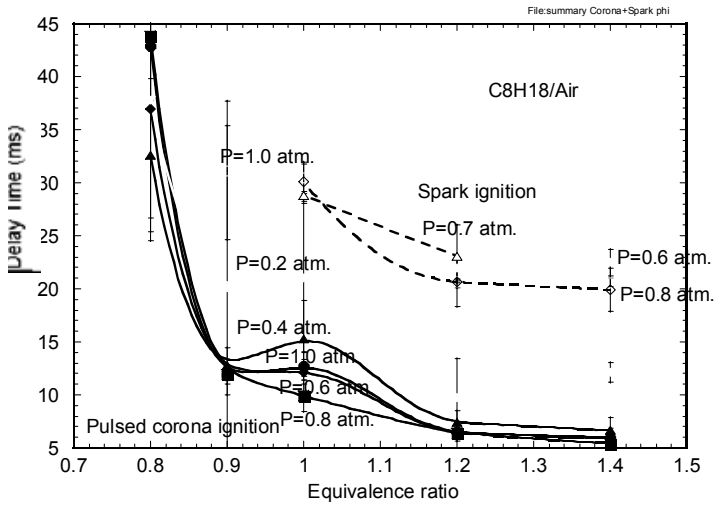

Fig.6 Delay time versus equivalence ratio of iso- $\mathrm{C}_{8} \mathrm{H}_{18} /$ Air

Iso-octane-air mixtures showed behavior similar to the other fuels. As shown in Figs. 5 and 6, over a wide range of equivalence ratio (0.8-1.4) and initial pressures (0.2-1.0 atm), the delay time and the rise time of pulsed corona ignited flames are shorter than that of spark ignited flames.

To compare ignition behavior between pulsed corona and spark discharges more explicitly, an improvement factor of delay time (rise time) was defined as the ratio between delay times (rise times) of flame ignited by spark discharge and pulsed corona discharge. For methane-air mixture, the average values of improvement factors of delay time and rise time over equivalence ratio range of 0.7-1.2 are 3.0 and 3.8, respectively. For iso-octane-air, they are 2.5 and 2.4 , respectively, over equivalence ratio range of 0.9 to 1.4 . 
Low pressure ignition limit, defined as the lowest pressure under which flame is ignitable in our particular pulsed corona discharge ignition device, for both methane-air and iso-octane-air, are $0.1 \mathrm{~atm}$ in the stoichiometric case and varies from 0.1atm. to $0.4 \mathrm{~atm}$. in equivalence ratio range from 0.7 to 1.4 for methane-air or from $0.1 \mathrm{~atm}$. to $0.2 \mathrm{~atm}$. in equivalence ratio range from 0.8 to 1.4 for isooctane-air.(Fig. 7). That might be of interest for high altitude relight applications.

\section{DISCUSSION}

Experiments show that corona discharge ignition provides shorter (by typically $3 \mathrm{x}$ ) delay and rise times than flames ignited by spark discharge, even at the most favorable spark location. There are at least two possible reasons: geometrical and chemical.

Geometrical advantages of pulsed corona ignition probably exist because pulsed corona discharge creates several hundred discharge channels filling the chamber volume compared to one unnecessarily intense channel for spark. If a significant fraction of these channels produce successful ignition kernels, the distance and time each kernel must travel to consume its share of combustible mixture is greatly reduced compared to single spark, thus delay and rise times are decreased.

Another possible advantage of corona discharges is, as mentioned earlier, the probable higher initial concentration of radicals. As shown in Fig. 2a., in case of identical ignition site number and energy, pulsed corona discharge produce significantly shorter delay time than spark discharge. That might be due to the higher radical producing efficiency of high energy electrons of corona discharge. This chemical factor mainly affect delay time but not rise time. Additionally, n-butane and iso-butane rise times (Fig. 4) follow the trends of their respective spark comparisons - even though the trends are different for the two fuels. This also indicates minor special chemical influence of corona discharges.

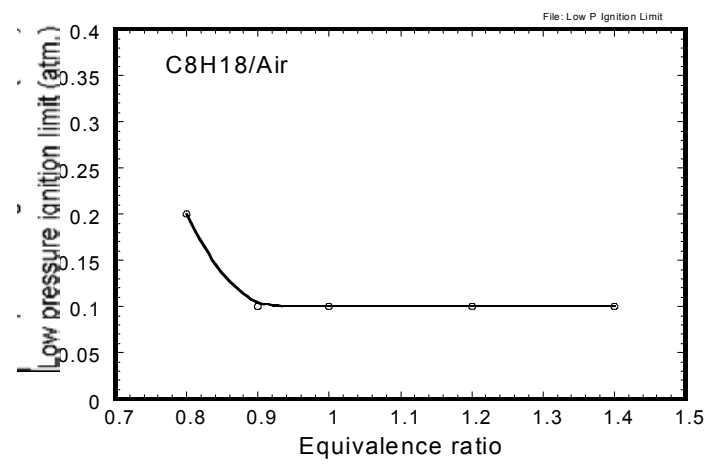

Fig. 8 Low ignition limit versus equivalence ratio of iso- $\mathrm{C}_{8} \mathrm{H}_{18} /$ Air.

The existence of "optimal energy", above which rise time reaches its shortest value and nearly independent of pulse energy and below which it is long and scatter could be explained as follows. Experiments show that the number of channels is relatively independent of pulse energy. A "minimum streamer energy" (or perhaps minimum energy per unit length) for ignition probably exists, analogous minimum ignition energies for conventional spark. At low energies only a few of these channels contain enough energy to initiate successful flame kernels. Therefore rise time tends to be longer and the values of the rise times are scatter because the locations of these discharge channels change irregularly. With increasing pulse energy, more channels can initiate kernels, causing shorter and shorter rise time. Above some pulse energy, most channels (or perhaps a constant fraction of channels) can initiate kernels, thus further pulse energy increases have little effect on rise time, and rise time values become stable.

These results suggest the following simple mechanism of corona ignition. Corona discharges yield shorter rise times by creating more distributed ignition sites within the gas. High energy electrons of corona discharge produce more radicals resulting shorter delay time. For fixed geometry, the streamer pattern is nearly independent of pulse energy. Rise times are determined primarily by the fraction of streamers containing sufficient energy for ignition. With low energy, only a few discharge channels have enough energy density to ignite flame and their location is irregular, resulting longer and scatter rise time values. With optimum energy, the number of discharge channels which are capable to ignite flame is 
large enough that further increasing of this number will not shorten or stable rise time further. High electron energy of corona discharge produces more radicals resulting shortening of delay time.

The authors would like to express their sincere thanks to ONR (Grant N00014-00-10098), AFOSR (Grant F49620-01-1-0322), ARO (Grant DAAD19-01-1-0698) and DOE (Grant DE-FC26-02NT41336) for their support.

* Author to whom correspondence should be directed: Martin A. Gundersen, Department of Electrical Engineering-Electrophysics, University of Southern California, Los Angeles, CA 90089-0271mag@usc.edu

\section{REFERENCES}

[1] Ronney, P.D "Laser versus conventional ignition of flames", Optical Engineering, Vol.33, 510-521 (1994).

[2] Phuoc, T.X., White, F.P., "Laser-induced spark ignition of CH4/Air mixtures" Combust. Flame Vol.119, 203-216 (1999).

[3] Jian-Bang Liu, Paul D. Ronney and Martin A. Gundersen, "Premixed flame ignition by transient plasma discharges", Proceedings of the Third Joint Meeting of the U.S. Sections, The Combustion Institute, Paper B-25, 2003 March 16-19, Chicago, Illinois

[4] JianBang Liu, Paul D. Ronney, Fei Wang, L.C. Lee and Martin Gundersen, "Transient plasma ignition for lean burn application", 41st Aerospace Sciences Meeting, No. 2003-6208 (2003), Reno, Nevada, 6 - 9 Jan 2003
[5] J.B.Liu, P.D.Ronney and M.A.Gundersen, "Premixed Flame Ignition by Transient, Plasma Discharges", $29^{\text {th }}$ Symposium (International) on Combustion, Combustion Institute, July 21 - 26, 2002, Sapporo, Japan.

[6] J.B.Liu, P. D. Ronney and M. A. Gundersen, "Premixed flame ignition by pulsed corona discharges", Spring 2001 meeting, Western States Section, the Combustion Institute, San Diego, La Jolla, CA.

[7] R. Maly, "Spark Ignition: Its physics and Effect on the Internal Combustion Engine", In "Fuel Economy: Road Vehicles Powered by Spark Ignition Engines" ed. by Hilliard, J.C. and Springer, G.S., Plenum Press, NY,1983, 91-148

[8] R. Maly, and M. Vogel, "Initiation and propagation of flame front in lean $\mathrm{CH}_{4}$-Air mixtures by the three models of the ignition spark", Proceedings of the $17^{\text {th }}$ Symposium (International) on Combustion, the Combustion Institute, Vol. 17, 821-831 1978

[9] N.B. Anikin, E.I. Mintoussov, S.V. Pancheshnyi, D.V. Roupassov, V.E. Sych, and A.Yu Starikovskii, "Nonequilibrium plasma and its applications for combustion and hypersonic flow control", ", 41st Aerospace Sciences Meeting, Reno, Nevada, 6 - 9 Jan 2003

[10] S.M. Bozhenkov, S.M. Starikovskaia, V.A.Sechenov, A.Yu Starikovskii, and V.P. Zhukov, "Combustion mixtures ignition in a wide pressure range. Nanosecond high-voltage discharge ignition", ", 41st Aerospace Sciences Meeting, Reno, Nevada, 6 - 9 Jan 2003

[11] Lewis, B., von Elbe, G., Combustion, Flames, and Explosions of Gases, 3rd Ed., Academic Press, 1987. 